\title{
Targeted Therapies in HER2-Overexpressing Metastatic Breast Cancer
}

\author{
Soumaya Labidi Nesrine Mejri Aymen Lagha Nouha Daoud Houda El Benna Mehdi Afrit \\ Hamouda Boussen
}

Medical oncology department, Abderrahmane Mami Hospital, University of medicine Tunis-University El Manar, Ariana, Tunisia

\section{Keywords \\ Metastatic breast cancer - Targeted therapy . HER2}

\section{Summary}

Human epidermal growth factor receptor-2 (HER2) is amplified in $25-30 \%$ of breast cancers and is associated with aggressive disease and poorer survival. Multiple anti-HER2 targeted therapies have dramatically changed management and outcome of this subgroup, both in adjuvant and metastatic settings. Despite the improvement of survival thanks to trastuzumab, unclear mechanisms of resistance occur, which has led to the development of new anti-HER2 therapies such as lapatinib, pertuzumab, and trastuzumab emtansine (T-DM1). The optimal sequence of the available drugs is still not well established. All this progress raises the question of toxicity that need to be managed, especially with longer survival of patients. In this article, we review different anti-HER2 therapies used in HER2-positive m etastatic breast cancer.

(C) 2016 S. Karger GmbH, Freiburg

\section{Introduction}

Breast cancer accounts for $25 \%$ of all cancers worldwide with more than 1 million new cases diagnosed each year, $5 \%$ being metastatic at presentation [1]. Analysis of signaling pathways led to the identification a human epidermal growth factor receptor-2 (HER2) overexpression subgroup, accounting for $15-20 \%$ of cases [2]. This overexpression is an adverse prognostic indicator of progressionfree survival (PFS) and overall survival (OS). Several murine mon- oclonal antibodies against the extracellular domain of the HER2 protein were found to inhibit the proliferation of human cancer cells that overexpress HER2 [3]. Trastuzumab was the first antiHER2 targeted agent found to increase PFS and OS among patients with metastatic disease. However, approximately $70 \%$ of patients become resistant to trastuzumab within a year [4]. There is no established biomarker to predict individuals who will becomes resistant. This led to the development of new anti-HER2 agents: lapatinib, pertuzumab and trastuzumab emtansine (T-DM1). Each of these drugs showed efficacy in different settings with longer survival in HER2-positive metastatic breast cancer (MBC), which raises the question of toxicity. Cardiac toxicity represents the major limiting factor. The aim of this review was to report the efficacy and safety profile of different anti-HER 2 targeted therapies available for MBC.

\section{Methods}

We searched for clinical trials published in PubMed, Medline, Cancerlit, Embase, Ovid online, Wiley online and Cochrane relevant to our review. Articles, abstracts and review articles were identified and reviewed. The reference lists from these sources were searched for additional trials. Only studies fully published in peer-reviewed journals were considered.

\section{Results}

\section{Trastuzumab}

Trastuzumab is a monoclonal IgG1 antibody against the human epidermal growth factor. It has shown efficacy in all lines as a single agent or in combination with chemotherapy, endocrine therapy or other anti-HER2 drugs. The first phase III clinical trial demonstrated an improvement in PFS (7.4 vs. 4.6 months; $\mathrm{p}<0.001$ ) and

\section{KARGER}

() 2016 S. Karger GmbH, Freiburg

Fax +497614520714 
OS (25.1 vs. 20.3 months; $\mathrm{p}=0.01$ ) [5]. Several chemotherapy drugs were tested in combination with trastuzumab: taxanes [6], capecitabine [7], vinorelbine [8] and gemcitabine [9] with few studies comparing those drugs head to head. Weekly paclitaxel is the most commonly used regimen, showing a high efficacy and low toxicity [10]. Recently, the HERNATA randomized clinical trial (RCT) compared the combination trastuzumab-docetaxel versus trastuzumab-vinorelbine in first line. No difference was seen in terms of PFS and OS; however, lower grade $3 / 4$ hematological toxicity was seen in the vinorelbine arm (36.0\% vs. $10.1 \%)$ [11]. Concomitant administration of trastuzumab and chemotherapy was superior to sequential schedule in terms of response rate (RR), PFS and OS in 3 RCTs [12].

As yet, no best endocrine therapy combination has been established. The TAnDEM study was the first RCT to demonstrate an improvement in PFS (4.8 vs. 2.4 months, $\mathrm{p}<0.001$ ) favoring the combination anastrozole-trastuzumab with a trend to a nonsignificant OS improvement [13]. The eLEcTRA trial showed that the combination of letrozole and trastuzumab is a safe and effective treatment option [14]. Several conflicting data suggest a complex cross-talk between HER-2 and the estrogen receptor, and it has been hypothesized that HER-2-positive tumors may be less responsive to certain endocrine treatments. A possible explanation for those results may be a relative resistance to endocrine therapy under an HER2 blockade [15]. Trastuzumab monotherapy is a feasible strategy in oligometastatic patients, with RRs ranging from 15 to $26 \%$ and a reported median time to treatment failure of 9 months [16]. Optimal treatment duration is still unclear. In the absence of evidence-based data, stopping chemotherapy and continuing trastuzumab alone until disease progression, is a reasonable option in patients showing maximum objective response or unacceptable toxicity. Beyond progression, trastuzumab with second-line capecitabine showed a better objective RR (27 vs. $48 \%$ ) and PFS (5.6 vs. 8.2 months, $\mathrm{p}=0.0338$ ) than capecitabine alone [17].

Cardiac toxicity remains the major concern with trastuzumab. Reduction in left ventricular ejection fraction is reversible and is not dose dependent [18]. It is observed in $27 \%$ of cases when used with anthracyclines, $13 \%$ with paclitaxel and in $3-7 \%$ when used alone [19]. HER2 signaling pathway blockade is responsible for cardiomyocyte growth, repair and survival alterations [20]. A proposed cardiotoxicity mechanism is loss of HER2-mediated signaling, which results in reduced ability of the heart to respond to stress [21]. Underlying cardiac risk factors are also believed to be important factors that may influence the expression of cardiac injury. However, such a mechanism is still under investigation. Synchronous toxicity with anthracycline-induced cardiac toxicity has led to this combination not being recommended. A recently published meta-analysis demonstrated that concomitant administration was moderately associated with increased risk of cardiac-related adverse events $(\mathrm{RR}=1.97,95 \%$ confidence interval $(\mathrm{CI}) 1.49$ $2.60, \mathrm{p}<0.001)$ [22]. Other minor toxicities such as allergic reactions and pneumopathy have been reported [23]. Overall, trastuzumab is considered a well-tolerated treatment.

\section{Lapatinib}

Lapatinib is an orally available small molecule that inhibits the tyrosine kinases of HER2 and epidermal growth factor receptor type 1.2 pilot studies have shown efficacy of lapatinib-capecitabine in MBC: Geyer et al. in 2006 [24] and Cameron et al. in 2008 [25]. Lapatinib and 3-weekly paclitaxel as first-line therapy resulted in an improvement of PFS (36.4 vs. 25.1 weeks, $p=0.005$ ), compared to paclitaxel alone [26]. However, lapatinib is not recommended as first-line therapy. The combination of lapatinib-weekly paclitaxel was evaluated in a phase II study, showing an RR of $77 \%$ during a median of 42.3 weeks [27]. No OS benefit was reported. The toxicity profile was similar in weekly and 3-weekly regimens except for neutropenia, which was seen more frequent in the weekly regimen (44 vs. $26 \%$ ) for all grades, but similar in grade 3 or 4 (26 vs. $21 \%$ ). A letrozole and lapatinib combination was tested in the first-line setting with a significant improvement in PFS and RR, with clinical benefit lasting more than 6 months [28].

Few data are as yet available about the management of antiHER2 therapies beyond progression on lapatinib and trastuzumab. In a retrospective study of 69 cases, retreatment with trastuzumab resulted in an RR of 31\%, median time of response of 8 months and a clinical benefit of $47 \%$ [29].

Lapatinib activity on brain metastases is a subject of controversy, experimental data showed that lapatinib can overcome the blood-brain barrier and is active on brain lesions [30]. No phase III data about lapatinib and brain metastases are available. The CEREBEL study compared the impact of trastuzumab-capecitabine versus lapatinib-capecitabine on the incidence of brain metastases as first relapse site [31]. An interim analysis reported a low number of brain metastases events, with no difference in the incidence observed in between the 2 groups. The reason for this observation may be the screening magnetic resonance imaging required upon study inclusion. The major toxicity concern for lapatinib is diarrhea, which has been reported in $50-85 \%$ of patients; in lower intensity, skin rash, nausea and pruritus are seen [32]. In a pooled analysis, grade 3-4 diarrhea was observed in $6 \%$ of cases with monotherapy, in $14 \%$ of cases when combined to capecitabine, and in $9 \%$ of cases when combined to taxanes [33]. In cases of moderate to severe diarrhea, or complicating features such as severe cramping, severe nausea and vomiting, fever or dehydration, lapatinib treatment should be stopped, and hospitalization with octreotide and/or antibiotics therapy may be needed [34]. No correlation between skin reactions and response was reported. Reported incidence of cardiac toxicity was $1.6 \%$ (grade 3-4 defined as cardiac events or left ventricular ejection fraction reduction more than 20\%) [35].

\section{Lapatinib-Trastuzumab}

Dual blockade can be a substantial advance for patients with HER2-positive MBC. Lapatinib dual kinase inhibition directed against EGFR and HER2 kinases combined with HER2 inhibition 
Table 1. Pilot studies of trastuzumab and lapatinib in MBC

\begin{tabular}{|c|c|c|c|c|c|c|}
\hline Trial & $\mathrm{n}$ & Arms & Setting & $\begin{array}{l}\text { Overall response } \\
\text { rate, \% }\end{array}$ & PFS, months & OS, months \\
\hline Slamon et al. [5] & 234 & $\begin{array}{l}\text { trastuzumab + chemotherapy vs. } \\
\text { chemotherapy }\end{array}$ & first line & 50 vs. $32^{*}$ & 7.4 vs. $4.6^{*}$ & 25.1 vs. $20^{*}$ \\
\hline $\begin{array}{l}\text { von Minckwitz et al. } \\
\text { [17] }\end{array}$ & 156 & $\begin{array}{l}\text { trastuzumab+capecitabine vs. } \\
\text { capecitabine }\end{array}$ & beyond first line & 48.1 vs. 27 & 8.2 vs. 5.6 & 25.5 vs. 20.4 \\
\hline Kaufman et al. [13] & 207 & $\begin{array}{l}\text { anastrozole +trastuzumab vs. } \\
\text { anastrozole }\end{array}$ & any line & 20 vs. ${ }^{\star} 7$ & 4.8 vs. $2.4^{*}$ & 28.5 vs. 23.9 \\
\hline Huober et al. [14] & 92 & letrozole +trastuzumab vs. letrozole & any line & 27 vs. $13^{*}$ & 14.1 vs. 3.3 & na \\
\hline Di Leo et al [26] & 579 & lapatinib+ paclitaxel vs. paclitaxel & first line & 63 vs. $38^{*}$ & 8.5 vs. $5.8^{*}$ & 24.0 vs. $19.8^{*}$ \\
\hline Cameron et al. [25] & 399 & $\begin{array}{l}\text { lapatinib capecitabine vs. capecit- } \\
\text { abine }\end{array}$ & second line & 32 vs. 17 & 5.5 vs. $4.2^{*}$ & 15.6 vs. 15.3 \\
\hline Blackwell et al. [37] & 296 & trastuzumab+lapatinib vs. lapatinib & $\begin{array}{l}\text { after trastuzumab } \\
\text { progression }\end{array}$ & 10.3 vs. $6.9^{*}$ & $\begin{array}{l}12 \text { vs. } \\
8.1 \text { weeks * }\end{array}$ & $\begin{array}{l}51.6 \text { vs. } \\
39 \text { weeks }\end{array}$ \\
\hline Johnston et al. [28] & 44 & letrozole + lapatinib vs. letrozole & first line & 28 vs. $15^{\star}$ & 8.2 vs. $3.0^{*}$ & 33.3 vs. 32.2 \\
\hline
\end{tabular}

through trastuzumab showed significantly enhanced activity in breast cancer cells. This may be explained by synergistic activity with HER2 receptor increased phosphorylation [36]. The EGF104900 trial compared the combination of trastuzumab-lapatinib against lapatinib alone in patients who had progressed on trastuzumab, and reported longer PFS (11.1 vs. 8.1 weeks, $\mathrm{p}=0.011$ ) and OS (14 vs. 9.5 months, $\mathrm{p}=0.026$ ) for the combination [37]. Toxicity was well balanced between both arms except for all grades of diarrhea (60 vs. $40 \%$ ); grade 3 or higher was similar in both arms (7\%). Cardiac toxicity was low in both arms (5.4 vs. $2.1 \%$ ). In another trial, results showed significantly improved PFS and clinical benefit rate for the combination therapy; RR and OS were similar in both arms [38] (table 1).

\section{Pertuzumab}

Pertuzumab is a humanized monoclonal antibody that binds HER2 at a different epitope of the HER2 extracellular domain (sub domain II). Pertuzumab prevents HER2 from dimerizing with other ligand-activated HER receptors, most notably HER3, this synergic action results in an improvement of anti-tumor activity. Pertuzumab as monotherapy or in combination with chemotherapy was not effective in a phase I/II clinical trial [39]. The Cleopatra study randomized patients, in a first-line setting ( $90 \%$ being trastuzumab naïve), between trastuzumab-pertuzumab-docetaxel and trastuzumab-docetaxel-placebo [40]. The results showed an improvement in PFS (median 18.5 vs. 12.4 months, $\mathrm{p}<0.001$ ) and OS (median 56.5 vs. 40.8 months). The adverse-events profile during the treatment period was balanced between the 2 groups, with an increase by $2 \%$ in the incidence of grade 3 or higher febrile neutropenia (13.8 vs. $7.8 \%)$. This was mainly observed in patients from Asia (26 vs. $12 \%$ ). Diarrhea (66.8 vs. $46.3 \%$ ) and skin rash (33.7 vs. $24.2 \%$ ) were reported in the Pertuzumab group. This combination became a standard of care in first-line MBC. The PHEREXA study is an ongoing phase II trial testing pertuzumab in combination with capecitabine, in second line, in patients who progressed on trastuzumab [41].

\section{Trastuzumab Emtansine (TDM-1)}

T-DM1 is an antibody drug conjugate that incorporates the HER2-targeted anti-tumor properties of trastuzumab with the cytotoxic activity of the microtubule-inhibitory agent DM1 (derivative of maytansine). T-DM1 allows intracellular drug delivery, thereby improving the therapeutic index and minimizing exposure of normal tissue. The EMILA trial comparing TDM1 and lapatinib-capecitabine showed an improvement of PFS (9.6 vs. 6.4 months, $\mathrm{p}<0.001)$ and OS (30.9 vs. 25.1 months, $\mathrm{p}<0.001)$ [41]. The MARIANNE trial demonstrated no inferiority in PFS between the TDM-1-containing regimen and trastuzumab-taxanes combination in further lines. Mature results are needed to make any conclusions [42]. The most common toxicities of T-DM1 in the EMILIA study were thrombocytopenia (12.9\%), and elevated serum concentrations of aspartate aminotransferase $(4.3 \%)$ and alanine aminotransferase (2.9\%). T-DM1 markedly inhibits megakaryocyte differentiation via a cytotoxic effect of DM1, with no direct effect on platelet activation or aggregation [43]. In the EMILIA study, cardiac toxicity was low; 8 patients $(1.7 \%)$ and 7 patients (1.6\%) in the TDM1 and lapatinib-capecitabine arms, respectively, had an ejection fraction that was less than $50 \%$ and at least 15 percentage points below the baseline value.

\section{Other Combinations}

\section{Bevacizumab}

HER2 overexpression is associated with upregulation of vascular endothelial growth factor (VEGF), which lead to the rationale 
behind a possible synergic activity of anti-HER2 therapy and antiVEGF therapy. The AVEREL study evaluated the addition of bevacizumab to trastuzumab-docetaxel in first-line setting in 424 MBC patients but could not show PFS improvement; there was, however, an increased grade $3 / 4$ febrile neutropenia and hypertension [44]. The combination lapatinib-bevacizumab was tested in a phase II study, which found an objective RR of $13 \%$, a clinical benefit beyond 24 weeks in $34 \%$ of cases, and a PFS at 12 weeks of $63 \%[45]$.

\section{Everolimus}

Everolimus is an orally administered inhibitor of the mammalian target of rapamycin (mTOR). The BOLERO-3 study randomized patients with HER2-positive, trastuzumab-resistant, advanced breast cancer to everolimus-trastuzumab-vinorelbine or placebo-trastuzumab-vinorelbine. Results showed that the addition of everolimus significantly prolonged PFS (7 vs. 5.8 months, $\mathrm{p}=0.006)$ [46]. The most common grade $3 / 4$ adverse events were neutropenia ( 73 vs. $62 \%$ ), leucopenia ( 38 vs. $29 \%$ ), anemia (19 vs. $6 \%)$ and febrile neutropenia (16 vs. $4 \%$ ). In the first-line setting, the recently presented BOLERO-1 trial did not show an improvement in PFS with the addition of everolimus to trastuzumab-paclitaxel (14.95 vs. 14.49 months, 95\% CI 14·55-17 ·91), except for the subgroup with negative hormone receptor status (20.27 vs. 13.08 months, $\mathrm{p}=0.0049$ ) [47].

\section{Perspective Combinations}

Neratinib is a potent, low-molecular-weight, orally administered, irreversible, pan-ErbR receptor tyrosine kinase inhibitor. Phase II studies assessed the safety and preliminary efficacy of the combination of neratinib plus trastuzumab [48] and paclitaxel [49], showing clinical activity (overall RR 27\%) and no significant toxicities.

Afatinib is a novel, potent, small-molecule tyrosine kinase inhibitor that irreversibly and selectively targets the HER2 receptors family. Several phase II studies designed to assess the efficacy and safety of afatinib in patients with HER2-positive MBC after failure of treatment with trastuzumab demonstrated a clinical activity with median PFS of 15.1 weeks and median OS of 61.0 weeks [50]. It also showed clinical activity when combined with vinorelbine.

\section{Recommendations and Sequence of Drug Use}

After the approval of these multiple anti-HER2 agents, new questions appeared mainly concerning the optimal sequence. There is now an urgent need for phase III randomized clinical trials addressing this particular question. European (ESMO) and American (NCCN) recommendations propose different options in different settings according to the available data [51, 52]. AntiHER2 therapy should be the treatment of choice in all patients with HER2-positive MBC. Combination with endocrine therapy or chemotherapy depends on hormone receptor status, endocrine sensitivity and clinical presentation. If endocrine therapy is considered, the addition of trastuzumab improves PFS without improving OS. If chemotherapy is considered, the preferred first-line therapy is trastuzumab-pertuzumab-taxane. Tratuzumab should be continued until progression (optimal duration is still unknown). After disease progression, several options are available. There are no data supporting the continuation of pertuzumab. Maintaining HER2 pathway suppression with trastuzumab continuation, lapatinib introduction or combining lapatinib with trastuzumab improved the course of the disease. If not used in first line, pertuzumab-trastuzumab chemotherapy can be used. The introduction of T-DM1 should be the treatment of choice after trastuzumab progression. T-DM1 is also the treatment of choice beyond second line. Country available therapies, contraindications and toxicities should be considered when indicating a treatment strategy.

\section{Conclusions}

The prognosis for HER2-overexpressing MBC has now improved thanks mainly to HER2 pathway inhibition using several agents. Translational studies trying to elucidate the different mechanisms of resistance may lead to clinically relevant therapies.

\section{Disclosure Statement}

This research did not receive any specific grants from funding agencies in the public, commercial, or not-for-profit sectors. The authors declare no conflict of interest.

\section{References}

1 Ferlay J, Steliarova-Foucher E, Lortet-Tieulent J, et al. Cancer incidence and mortality patterns in Europe: Estimates for 40 countries in 2012. Eur J Cancer 2013;49: 1374-1403.

2 Jemal A, Siegel R, Ward E, et al.: Cancer statistics, 2008. CA Cancer J Clin 2008;58:71-96.

$\checkmark 3$ Wolff A, Hammond ME, Hicks DG, et al.: Recommendations for human epidermal growth factor receptor 2 testing in breast cancer: AmericanSociety of Clinical Oncology/College of American Pathologists Clinical Practice Guideline Update. J Clin Oncol 2013;31: 3997-4013.
4 Slamon D, Clark G, Wong S, et al.: Human breast cancer: Correlation of relapse and survival with amplification of the HER-2/neuoncogene. Science 1987;235:177-182.

5 Slamon D, Leyland-Jones B, Shak S, et al.: Use of chemotherapy plus a monoclonal antibody against HER2 for metastatic breast cancer that overexpresses HER2. N Engl J Med 2001;344:783-792.

6 Seidman A, Berry D, Cirrincione C, et al.: Randomized phase III trial of weekly compared with every-3-weeks paclitaxel for metastatic breast cancer, with trastuzumab for all HER-2 overexpressors and random assignment to trastuzumab or not in HER-2 nonoverexpressors: Final results of Cancer and Leukemia Group B protocol 9840. J Clin Oncol 2008;26:1642-1649.
7 Bartsch R, Wenzel C, Altorjai G, et al.: Capecitabine and trastuzumab in heavily pretreated metastatic breast cancer. J Clin Oncol 2007;25:3853-3858.

8 Burstein H, Keshaviah A, Baron A, et al.: Trastuzumab plus vinorelbine or taxane chemotherapy for HER2overexpressing metastatic breast cancer: The trastuzumab and vinorelbine or taxane study. Cancer 2007; 110:965-972.

9 O'Shaughnessy J, Vukelja S, Marsland T, et al.: Phase II study of trastuzumab plus gemcitabine in chemotherapy-pretreated patients with metastatic breast cancer. Clin Breast Cancer 2004;5:142-147. 
10 Fountzilas G, Dafni U, Dimopoulos M, et al.: A randomized phase III study comparing three anthracycline-free taxane-based regimens, as first line chemotherapy, in metastatic breast cancer: A Hellenic Cooperative Oncology Group study. Breast cancer Res Treat 2009;115:87-89.

11 Andersson M, Lidbrink E, Bjerre K, et al.: Phase III randomized study comparing docetaxel plus trastuzumab with vinorelbine plus trastuzumab as first-line therapy of metastatic or locally advanced human epidermal growth factor receptor 2-positive breast cancer: The HERNATA study. J Clin Oncol 2011;29:264-271.

12 Hamberg P, Bos M, Braun H, et al.: Dutch Breast Cancer Trialists' Group (BOOG). Randomized phase II study comparing efficacy and safety of combinationtherapy trastuzumab and docetaxel vs. sequential therapy of trastuzumab followed by docetaxel alone at progression as first-line chemotherapy in patients with HER2+ metastatic breast cancer: HERTAX trial. Clin Breast Cancer 2011;11:103-113.

13 Kaufman B, Mackey J, Clemens M, et al.: Trastuzumab plus anastrozole versus anastrozole alone for the treatment of postmenopausal women with human epidermal growth factor receptor 2-positive, hormone receptor-positive metastatic breast cancer: results from the randomized phase III TAnDEM study. J Clin Oncol 2009;27:5529-5537.

14 Huober J, Fasching P, Barsoum M, et al.: Higher efficacy of letrozole in combination with trastuzumab compared to letrozole monotherapy as first-line treatment in patients with HER2-positive, hormone-receptor-positive metastatic breast cancer. Results of the eLEcTRA trial. Breast 2012;21:27-33.

15 Dowsett M: Overexpression of HER-2 as a resistance mechanism to hormonal therapy for breast cancer. Endocr Relat Cancer 2001;8:191-195.

16 Sawaki M, Ito Y, Tada K, et al.: Efficacy and safety of trastuzumab as a single agent in heavily pretreated patients with HER-2/neu-overexpressing metastatic breast cancer. Tumori 2004;90:40-43.

17 Von Minckwitz G, Du Bois A, Schmidt M, et al.: Trastuzumab beyond progression in human epidermal growth factor receptor 2-positive advanced breast cancer: A German breast group/breast international group 03-05 study. J Clin Oncol 2009;27:1999-2006.

18 Suter T, Procter M, van Veldhuisen D, et al.: Trastuzumab associated cardiac adverse effects in the herceptin adjuvant trial. J Clin Oncol 2007;25:3859-3865.

19 Seidman A, Hudis C, Pierri MK, et al.: Cardiac dysfunction in the trastuzumab clinical trials experience. J Clin Oncol. 2002;20:1215-1221.

20 Negro A, Brar B, Lee K: Essential roles of Her2/erbB2 in cardiac development and function. Recent Prog Horm Res 2004;59:1-12.

21 Keefe D: Trastuzumab-associated cardiotoxicity. Cancer 2002;95:1592-1600.

22 Du F, Yuan P, Zhu W, et al.: Is it safe to give anthracyclines concurrently with trastuzumab in neo-adjuvant or metastatic settings for HER2-positive breast cancer? A meta-analysis of randomized controlled trials. Med Oncol 2014;31:1-9.

23 Lenz HJ: Management and preparedness for infusion and hypersensitivity reactions. Oncologist 2007;12: 601-609.

24 Geyer C, Forster J, Lindquist D, et al.: Lapatinib plus capecitabine for HER2-positive advanced breast cancer. N Engl J Med 2006;355:2733-2743.
25 Cameron D, Casey M, Press M, et al.: A phase III randomized comparison of lapatinib plus capecitabine versus capecitabine alone in women with advanced breast cancer that has progressed on trastuzumab: Updated efficacy and biomarker analyses. Breast Cancer Res Treat 2008;112:533-543.

26 Di Leo A, Gomez H, Aziz Z, et al.: Phase III, doubleblind, randomized study comparing lapatinib plus paclitaxel with placebo plus paclitaxel as first-line treatment for metastatic breast cancer. J Clin Oncol 2008; 26:5544-5552.

27 Jagiello-Gruszfeld A, Tjulandin Sergei S, Dobrovols kaya N, et al.: Lapatinib (L) with weekly paclitaxel (P) as first-line therapy for patients (pts) with HER2+ metastatic breast cancer (MBC). San Antonio Breast Cancer Symposium 2008, 2008.

28 Johnston S, Pippen J Jr, Pivot X, et al.: Lapatinib combined with letrozole versus letrozole and placebo as first-line therapy for postmenopausal hormone receptor-positive metastatic breast cancer. J Clin Oncol 2009;27:5538-5546.

29 Gori S, Montemurro F, Spazzapan S, et al.: Retreatment with trastuzumab-based therapy after disease progression following lapatinib in HER2-positive metastatic breast cancer. Ann Oncol 2012;23:1436-1441.

30 Gril B, Palmieri D, Bronder JL, et al.: Effect of lapatinib on the outgrowth of metastatic breast cancer cells to the brain. J Natl Cancer Inst 2008; 100:1092-1103.

31 Pivot X, Semiglazov B, Zurawski B, et al.: CEREBEL (EGF111438): An open label randomized phase III study comparing the incidence of CNS metastases in patients (pts) with HER2+ metastatic breast cancer (MBC), treated with lapatinib plus capecitabine (LC) versus trastuzumab plus capecitabine (TC). Ann Oncol 2012;23(suppl 9):ixe5 abstract LBA11 doi:10.1093/ annonc/mds499.

32 Lacouture M, Laabs S, Koehler M, et al.: Analysis of dermatologic events in patients with cancer treated with lapatinib. Breast Cancer Res Treat 2009;114:485493.

33 Crown J, Burris H, Boyle F, et al.: Pooled analysis of diarrhea events in patients with cancer treated with lapatinib. Breast Cancer Res Treat 2008;112:317-325.

34 Benson A, Ajani J, Catalano R, et al.: Recommended guidelines for the treatment of cancer treatmentinduced diarrhea J Clin Oncol 2004;22:2918-2926.

35 Perez E, Koehler M, Byrne J, et al.: Cardiac safety of lapatinib: pooled analysis of 3689 patients enrolled in clinical trials. Mayo Clin Proc 2008;83:679-686.

36 Konecny G, Pegram M, Venkatesan N, et al.: Activity of the dual kinase inhibitor lapatinib (GW572016) against HER-2-overexpressing and trastuzumab-treated breast cancer cells. Cancer Res 2006;66;1630-1639.

37 Blackwell K, Burstein H, Storniolo A, et al.: Overall survival benefit with lapatinib in combination with trastuzumab for patients with human epidermal growth factor receptor 2-positive metastatic breast cancer: final results from the EGF104900 Study. J Clin Oncol 2012;30:2585-2592.

38 O'shaughnessy J, Blackwell K, Burstein H, et al.: A randomized study of lapatinib alone or in combination with trastuzumab in heavily pretreated HER2+ metastatic breast cancer progressing on trastuzumab therapy. J Clin Oncol 2008;26(15S):1015.
9 Cortés J, Fumoleau P, Bianchi GV, et al.: Pertuzumab monotherapy after trastuzumab-based treatment and subsequent reintroduction of trastuzumab: Activity and tolerability in patients with advanced human epidermal growth factor receptor 2-positive breast cancer. J Clin Oncol 2012;30:1594-1600.

40 Swain S, Kim S-B, Cortes J, et al.: Confirmatory overall survival (OS) analysis of CLEOPATRA: A randomized, double-blind, placebo-controlled phase III study with pertuzumab (P), trastuzumab (T), and docetaxel (D) in patients (pts) with HER2-positive first-line (1L) metastatic breast cancer (MBC). Cancer Res 2012;72:476s.

41 Capelan M, Pugliano L, De Azambuja E, et al.: Pertuzumab: New hope for patients with HER2-positive breast cancer. Ann Oncol 2013;24:273-282.

42 Ellis PA, Barrios CH, Eiermann W, et al.: Phase III, randomized study of trastuzumab emtansine (T-DM1) \pm pertuzumab $(\mathrm{P})$ vs trastuzumab+ taxane $(\mathrm{HT})$ for first-line treatment of HER2-positive MBC: Primary results from the MARIANNE study. J Clin Oncol 2015; 33 (suppl):abstr 507.

43 Uppal H, Doudement E, Mahapatra K, et al.: Potential mechanisms for thrombocytopenia development with trastuzumab emtansine (T-DM1). Clin Cancer Res 2015;21:123-133.

44 Gianni L, Romieu GH, Lichinitser M, et al.: AVEREL. A randomized phase III trial evaluating bevacizumab in combination with docetaxel and trastuzumab as first-line therapy for HER2-positive locally recurrent/ metastatic breast cancer. J Clin Oncol 2013;31:17191725.

45 Dickler M, Franco S, Stopeck A, et al.: Final results from a phase II evaluation of lapatinib (L) and bevacizumab (B) in HER2-overexpressing metastatic breast cancer (MBC). Cancer Res 2009;69(2 suppl):3133.

46 André F, O’Regan R, Ozguroglu M, et al.: Everolimus for women with trastuzumab-resistant, HER2-positive, advanced breast cancer (BOLERO-3): A randomised, double-blind, placebo-controlled phase 3 trial. Lancet Oncol 2014;15:580-591.

47 Hurvitz SA, Andre F, Jiang Z, et al.: Combination of everolimus with trastuzumab plus paclitaxel as firstline treatment for patients with HER2-positive advanced breast cancer (BOLERO-1): A phase 3, randomised, double-blind, multicentre trial. Lancet Oncol 2015;16:816-829.

48 Swaby R, Blackwell K, Jiang Z, et al.: Neratinib in combination with trastuzumab for the treatment of advanced breast cancer: A phase I/II study. J Clin Oncol 2009;27(15S):1004.

49 Chow L, Gupta S, Hershman D, et al.: Safety and efficacy of neratinib (HKI-272) in combination with paclitaxel in ErbB2+ metastatic breast cancer. Cancer Res 2009;69(suppl 3): 792s.

50 Lin N, Winer E, Wheatley D, et al.: A phase II study of afatinib (BIBW 2992), an irreversible ErbB family blocker, in patients with HER2-positive metastatic breast cancer progressing after trastuzumab. Breast Cancer Res Treat 2012;133:1057-1065.

51 Cardoso F, Harbeck N, Fallowfield L, et al.: Locally recurrent or metastatic breast cancer: ESMO Clinical Practice Guidelines for diagnosis, treatment and follow-up. Ann Oncol 2012;23(suppl 7):11-19.

52 Carlson R, Allred D, Anderson B, et al.: Metastatic breast cancer, version 1.2012: Featured updates to the NCCN guidelines. J Natl Compr Canc Netw 2012;10: 821-829. 